\title{
New Spherically Symmetric Solutions in $f(R)$-gravity by Noether Symmetries
}

\author{
Salvatore Capozziello ${ }^{1,2 *}$, Noemi Frusciante ${ }^{3,4 \dagger}$, Daniele Vernieri ${ }^{3,4 \ddagger}$ \\ ${ }^{1}$ Dipartimento di Scienze Fisiche, Università di Napoli "Federico II" and ${ }^{2}$ INFN Sez. di Napoli, \\ Compl. Univ. di Monte S. Angelo, Edificio G, Via Cinthia, I-80126, Napoli, Italy \\ ${ }^{3}$ SISSA-International School for Advanced Studies, \\ Via Bonomea 265, 34136 Trieste, Italy and ${ }^{4}$ INFN, \\ Sez. di Trieste, Via Valerio 2, 34127, Trieste, Italy
}

(Dated: September 12, 2018)

\begin{abstract}
Spherical symmetry for $f(R)$-gravity is discussed by searching for Noether symmetries. The method consists in selecting conserved quantities in form of currents that reduce dynamics of $f(R)$ models compatible with symmetries. In this way we get a general method to obtain constants of motion without setting a priori the form of $f(R)$. In this sense, the Noether symmetry results a physical criterium. Relevant cases are discussed.

Keywords: alternative theories of gravity; exact solutions; Noether symmetries
\end{abstract}

\section{INTRODUCTION}

The issue to renormalize General Relativity (GR) leads to effective theories of gravity including corrections in the curvature invariants to the Einstein-Hilbert action [1 $[4]$. In addition, the discovery that the expansion of the universe is accelerated [5] has led to the introduction of the so-called dark energy to explain the accelerated expansion of the Hubble fluid which, up to now, has no agreed interpretation at fundamental level.

In order to avoid this ad hoc ingredient, it has been proposed [6] that, perhaps, one needs to modify GR at the cosmic scales to address the observational evidences.

In particular, $f(R)$-gravity, where $f$ is a function of the Ricci scalar $R$ (in particular an analytic function), has been considered to explain the present cosmic acceleration without dark energy (see [7-10] for reviews). Here we focus on the metric version of these theories, where the field equations are of fourth order for the metric.

The full investigation of such theory is the first step toward the so-called Extended Theories of Gravity where effective actions involving generic curvature invariants are taken into account [11-15]. The goal is to address, by gravitational sector, problems like dark energy and dark matter at infrared scales and renormalization at ultraviolet scales.

In this view, exact solutions of the field equations are important in order to gain insight into the mathematical and physical content of a theory [16]. They can be obtained only upon assuming special symmetries. In the case of metric $f(R)$-gravity in vacuo, assuming spherical symmetry does not automatically lead to the Schwarzschild or Schwarzschild-de Sitter solutions [17]. The latter do solve the $f(R)$ vacuum field equations, but the Birkhoff theorem of GR does not hold in metric $f(R)$ gravity (see [18, 19] for a detailed discussion) and, therefore, these are not all the solutions within the family of spherically symmetric solutions [20 23]. In fact, the class of spherically symmetric solutions of metric $f(R)$-gravity is still unexplored, with only a handful of analytical solutions beyond Schwarzschildde Sitter being known. When one considers axially symmetric solutions, the situation is even worse: apart from the Kerr metric [24], only the solutions of Ref. 25] are presently available.

Because of the fact that these models have fourth order field equations, it is usually difficult to find exact solutions in full generality without imposing a-priori a particular form of the $f(R)$ function. To this aim, it is possible to consider spherically symmetric background and demonstrate that it is possible to find exact solutions via the Noether Symmetry Approach [19, 26 28]. In fact, choosing an appropriate $f(R)$-Lagrangian, it is possible to find out conserved Noether currents which will be useful to solve dynamics. This approach is very powerful due to the fact that it allows to find a closed system of equations, where we do not need to impose the particular form of the $f(R)$ which, on the other hand, is selected by the Noether symmetry itself. In Section II we introduce the $f(R)$-gravity using a suitable Lagrangian approach in the hypothesis of a spherically symmetric metric. Dynamical equations are solved in the case of constant curvature recovering the standard case of the Schwarzschild-de Sitter solution. In Section III we describe, in general, the Noether Symmetry Approach and in Section IV] we apply it to spherically symmetric $f(R)$-gravity.

\footnotetext{
* e-mail address: capozziello@na.infn.it

$\dagger$ e-mail address: noemi.frusciante@sissa.it

$\ddagger$ e-mail address: daniele.vernieri@sissa.it
} 
In Section $\mathrm{V}$ we find solutions to the dynamics for both constant curvature and in the hypothesis of a static metric. Conclusions are given in Section VI.

\section{SPHERICALLY SYMMETRY IN $f(R)$ GRAVITY}

Let us start with a generalization of the Hilbert-Einstein action

$$
\mathcal{S}=\int d^{4} x \sqrt{-g} f(R)
$$

where $f(R)$ is an analytic function of the Ricci scalar $R$ and $g$ is the determinant of the metric $g_{\mu \nu}$. We consider a time dependent spherically symmetric metric

$$
d s^{2}=e^{\nu(r, t)} d t^{2}-e^{\lambda(r, t)} d r^{2}-r^{2} d \Omega^{2}
$$

where $\nu(r, t)$ and $\lambda(r, t)$ are unknown functions of $r$ and $t$ and $d \Omega^{2}=\left(d \theta^{2}+\sin ^{2} \theta d \phi^{2}\right)$, using unit of $c=1$. In the following equations, we omit the $t$ and $r$ dependence, to avoid burdening the text. The Ricci scalar can be written as

$$
R=-\frac{2}{r^{2}}+e^{-\lambda}\left[\nu_{r r}+\frac{1}{2} \nu_{r}\left(\nu_{r}-\lambda_{r}\right)+\frac{2}{r}\left(\nu_{r}-\lambda_{r}\right)+\frac{2}{r^{2}}\right]+e^{-\nu}\left[\frac{1}{2} \lambda_{t}\left(\nu_{t}-\lambda_{t}\right)-\lambda_{t t}\right],
$$

where the subscripts $r$ and $t$ indicate radial and time partial derivatives. In order to obtain a canonical Lagrangian, function of $\nu(r, t), \lambda(r, t)$ and their first derivatives, we use the method of Lagrange multipliers [17, 29, 30]. Let us introduce the variable $\xi$ which is the Lagrange multiplier: this allows to set the Ricci scalar $R$ as a constraint for the dynamics. In this way the point-like action can be written as

$$
\tilde{\mathcal{S}}=\int d r d t r^{2} e^{\frac{\nu+\lambda}{2}}\left\{f(R)-\xi\left[R+\frac{2}{r^{2}}-e^{\lambda}\left[\nu_{r r}+\frac{1}{2} \nu_{r}\left(\nu_{r}-\lambda_{r}\right)+\frac{2}{r}\left(\nu_{r}-\lambda_{r}\right)+\frac{2}{r^{2}}\right]-e^{-\nu}\left[\frac{1}{2} \lambda_{t}\left(\nu_{t}-\lambda_{t}\right)-\lambda_{t t}\right]\right]\right\} .
$$

By this approach, we can consider $R(r, t), \nu(r, t)$ and $\lambda(r, t)$ as independent Lagrangian variables. The Lagrange multiplier can be easily found by varying the action with respect to $R$, and the result is

$$
\xi=f^{\prime}(R)
$$

where the prime denotes the derivative with respect to $R$. Using this expression for $\xi$ and integrating by parts, action (4) can be rewritten as

$$
\tilde{\mathcal{S}}=\int d t d r r^{2} e^{\frac{\nu+\lambda}{2}}\left\{f(R)-R f^{\prime}(R)-2 \frac{f^{\prime}(R)}{r^{2}}+e^{-\lambda}\left[2 \frac{f^{\prime}(R)}{r^{2}}-2 \frac{f^{\prime}(R)}{r} \lambda_{r}-f^{\prime \prime}(R) R_{r} \nu_{r}\right]+e^{-\nu} f^{\prime \prime}(R) R_{t} \lambda_{t}\right\} .
$$

Clearly, action (6), when varied with respect to $R, \nu$, and $\lambda$, gives the same equations of (11). Furthermore, we are disregarding a divergence and this fact could affect the conserved current. Finally, we are going to consider internal (i.e. vertical) transformations so that it is not important to know the transformation rules of $\nu$ and $\lambda$ with respect to spacetime transformations. The equations of motion are

$$
\begin{aligned}
& -\frac{2}{r} f^{\prime \prime}(R) R_{r}+\frac{1}{2} f^{\prime \prime}(R) \lambda_{r} R_{r}-f^{\prime \prime \prime}(R) R_{r}^{2}-f^{\prime \prime}(R) R_{r r}+\frac{1}{r} f^{\prime}(R) \lambda_{r}-\frac{1}{r^{2}} f^{\prime}(R)+ \\
& +e^{\lambda}\left[\frac{1}{2} R f^{\prime}(R)-\frac{1}{2} f(R)+\frac{1}{r^{2}} f^{\prime}(R)\right]+\frac{1}{2} e^{-\nu} f^{\prime \prime}(R) R_{t} \lambda_{t}=0
\end{aligned}
$$

for $\lambda$ and 


$$
\begin{aligned}
& e^{-\nu}\left[-\frac{1}{2} f^{\prime \prime}(R) \nu_{t} R_{t}+f^{\prime \prime \prime}(R) R_{t}^{2}+f^{\prime \prime}(R) R_{t t}\right]-\frac{1}{r^{2}} f^{\prime}(R)-\frac{1}{r} f^{\prime}(R) \nu_{r}-\frac{2}{r} f^{\prime \prime}(R) R_{r}-\frac{1}{2} f^{\prime \prime}(R) R_{r} \nu_{r}+ \\
& +e^{\lambda}\left[\frac{1}{2} R f^{\prime}(R)-\frac{1}{2} f(R)+\frac{1}{r^{2}} f^{\prime}(R)\right]=0
\end{aligned}
$$

for $\nu$. The equation for $R(r, t)$ is given by Eq. (3), that is the Euler constraint on the dynamics. Finally, we obtain a system of three partial differential Eqs. (17), (8) and (3) ) in the unknown functions $\nu(r, t), \lambda(r, t)$ and the constraint $R(r, t)$, once $f(R)$ is given. As a first check it is easy to see that Eqs. (7) and (8) allow us to recover the well-known results of GR by replacing $f(R)=R$.

It is straightforward to show that when curvature is constant over space and time $\left(R=R_{0}\right)$, an interesting solution can be found. Indeed subtracting Eqs. (7) and (8) we immediately obtain

$$
\lambda_{r}+\nu_{r}=0
$$

and substituting into Eq. (7), the solution for the metric component $g_{r r}$ is

$$
e^{\lambda(r, t)}=\frac{1}{1-\frac{\Lambda r^{2}}{3}-\frac{A_{1}(t)}{2 r}}
$$

where $A_{1}(t)$ is an integration function depending on time, and

$$
\Lambda=-\frac{1}{2}\left[R_{0}-\frac{f\left(R_{0}\right)}{f^{\prime}\left(R_{0}\right)}\right]
$$

Solving Eq. (9), we get

$$
e^{\nu(r, t)}=A_{2}(t)\left[1-\frac{\Lambda r^{2}}{3}-\frac{A_{1}(t)}{2 r}\right]=A_{2}(t) e^{-\lambda(r, t)},
$$

where $A_{2}(t)$ is another integration function depending on time. Inserting Solutions (10) and (12) into Eq. (3), we have a constraint on the integration functions: it is easy to show that the only way to make Eq. (3) consistent is to choose $A_{1}(t)=A_{1}=$ constant, for which we recover the well-known static Schwarzschild-De Sitter solution

$$
d s^{2}=\left[1-\frac{\Lambda r^{2}}{3}-\frac{A_{1}}{2 r}\right] d \tilde{t}^{2}-\frac{1}{1-\frac{\Lambda r^{2}}{3}-\frac{A_{1}}{2 r}} d r^{2}-r^{2} d \Omega^{2}
$$

where time as been scaled accordingly

$$
d \tilde{t}^{2}=A_{2}(t) d t^{2}
$$

being $R_{0}=-4 \Lambda$. The metric is time independent, and we have thus proved the Birkhoff theorem in the case of $f(R)$-gravity with constant curvature. It is worth noticing that such a theorem does not hold in general for any $f(R)$-model as shown in [13, 19].

\section{NOETHER SYMMETRY APPROACH}

Let us consider now the Noether Symmetry Approach by which it is possible to obtain conserved quantities asking for the invariance of the Lagrangian under the Lie derivative along an appropriate vector field. In this way, constraints on dynamics are achieved and we it is possible to solve the equations of motion. In general, a point-like canonical Lagrangian $\mathcal{L}$ depends on the variables $q^{j}\left(x^{\mu}\right)$ and on their derivatives $\partial_{\nu} q^{j}\left(x^{\mu}\right)$. The corresponding Euler-Lagrange equations are 


$$
\partial_{\mu} \frac{\partial \mathcal{L}}{\partial \partial_{\mu} q^{j}}=\frac{\partial \mathcal{L}}{\partial q^{j}} .
$$

Contracting eq. (14) with some unknown functions $\alpha^{j}=\alpha^{j}\left(q^{i}\right)$, it gives

$$
\alpha^{j}\left(\partial_{\mu} \frac{\partial \mathcal{L}}{\partial \partial_{\mu} q^{j}}-\frac{\partial \mathcal{L}}{\partial q^{j}}\right)=0 .
$$

Since we can write

$$
\alpha^{j} \partial_{\mu} \frac{\partial \mathcal{L}}{\partial \partial_{\mu} q^{j}}=\partial_{\mu}\left(\alpha^{j} \frac{\partial \mathcal{L}}{\partial \partial_{\mu} q^{j}}\right)-\left(\partial_{\mu} \alpha^{j}\right) \frac{\partial \mathcal{L}}{\partial \partial_{\mu} q^{j}},
$$

from Eq. (15) we immediately obtain

$$
\partial_{\mu}\left(\alpha^{j} \frac{\partial \mathcal{L}}{\partial \partial_{\mu} q^{j}}\right)=\alpha^{j} \frac{\partial \mathcal{L}}{\partial q^{j}}+\left(\partial_{\mu} \alpha^{j}\right) \frac{\partial \mathcal{L}}{\partial \partial_{\mu} q^{j}}=L_{\mathbf{X}} \mathcal{L},
$$

where $L_{\mathbf{X}}$ denotes the Lie derivative along the vector field

$$
\mathbf{X}=\alpha^{j} \frac{\partial}{\partial q^{j}}+\left(\partial_{\mu} \alpha^{j}\right) \frac{\partial}{\partial \partial_{\mu} q^{j}},
$$

which represent the generator of symmetry. We can immediately infer the Noether Theorem which states that if $L_{\mathbf{X}} \mathcal{L}=0$, the Lagrangian $\mathcal{L}$ is invariant along the vector field $\mathbf{X}$. As a consequence, we can define the current [31]

$$
j^{\mu}=\alpha^{j} \frac{\partial \mathcal{L}}{\partial \partial_{\mu} q^{j}},
$$

which is conserved being

$$
\partial_{\mu} j^{\mu}=0 .
$$

As already developed in [34], the presence of Noether symmetries allows to reduce dynamics and then to find out exact solutions. Specifically, symmetries select also the analytic form of $f(R)$. A similar approach has been developed for cosmological solutions [27].

\section{NOETHER SYMMETRIES IN SPHERICALLY SYMMETRIC $f(R)$ GRAVITY}

Let us take into account the case of spherical-symmetry in $f(R)$-gravity. For the sake of generality, we can assume that the vector field $\mathbf{X}$ depends on the whole set of configuration variables that are functions of the radial and time coordinates. The configuration space is $\mathcal{Q}=\{\nu(r, t), \lambda(r, t), R(r, t)\}$, so that the generator of symmetry ${ }^{1}$ becomes:

$$
\mathbf{X}=\alpha \frac{\partial}{\partial \nu}+\beta \frac{\partial}{\partial \lambda}+\gamma \frac{\partial}{\partial R}+\alpha_{r} \frac{\partial}{\partial \nu_{r}}+\beta_{r} \frac{\partial}{\partial \lambda_{r}}+\gamma_{r} \frac{\partial}{\partial R_{r}}+\alpha_{t} \frac{\partial}{\partial \nu_{t}}+\beta_{t} \frac{\partial}{\partial \lambda_{t}}+\gamma_{t} \frac{\partial}{\partial R_{t}},
$$

where $\alpha, \beta$ and $\gamma$ are functions of the variables $\nu, \lambda$ and $R$. As discussed above, a symmetry exists if the equation $L_{\mathbf{X}} \mathcal{L}=0$ has solutions for the functions $\alpha, \beta$ and $\gamma$ where at least one of them is different from zero. To verify such

\footnotetext{
${ }^{1}$ It is worth noticing that $R$, due to the constraint Eq. (3), is not a proper variable. However, it is needed in order to make canonical the Lagrangian.
} 
a condition, we can set to zero the coefficients of terms $\nu_{r}^{2}, R_{r}^{2}, \lambda_{t}^{2}, R_{t}^{2}, \nu_{r} \lambda_{r}, \nu_{r} R_{r}, \lambda_{r} R_{r}, \nu_{t} \lambda_{t}, \nu_{t} R_{t}$ and $\lambda_{t} R_{t}$ in $L_{\mathbf{X}} \mathcal{L}=0$ [32]. We obtain the following system of partial differential equations, linear in $\alpha, \beta$ and $\gamma$ :

$$
\begin{aligned}
& -r^{2} e^{\frac{\nu-\lambda}{2}} f^{\prime \prime}(R) \frac{\partial \gamma}{\partial \nu}=0 \\
& -r^{2} e^{\frac{\nu-\lambda}{2}} f^{\prime \prime}(R) \frac{\partial \alpha}{\partial R}=0, \\
& r^{2} e^{\frac{\lambda-\nu}{2}} f^{\prime \prime}(R) \frac{\partial \gamma}{\partial \lambda}=0, \\
& r^{2} e^{\frac{\lambda-\nu}{2}} f^{\prime \prime}(R) \frac{\partial \beta}{\partial R}=0 \\
& -r^{2} e^{\frac{\nu-\lambda}{2}} f^{\prime \prime}(R) \frac{\partial \gamma}{\partial \lambda}=0 \\
& \frac{\alpha}{2}+\frac{\partial \alpha}{\partial \nu}-\frac{1}{2} \beta+\frac{f^{\prime \prime \prime}(R)}{f^{\prime \prime}(R)} \gamma+\frac{\partial \gamma}{\partial R}=0, \\
& -r^{2} e^{\frac{\nu-\lambda}{2}} f^{\prime \prime}(R) \frac{\partial \alpha}{\partial \lambda}=0, \\
& r^{2} e^{\frac{\lambda-\nu}{2}} f^{\prime \prime}(R) \frac{\partial \gamma}{\partial \nu}=0 \\
& r^{2} e^{\frac{\lambda-\nu}{2}} f^{\prime \prime}(R) \frac{\partial \beta}{\partial \nu}=0, \\
& \frac{1}{2} e^{\nu-\lambda}(\beta-\alpha)+\frac{\partial \beta}{\partial \lambda}+\gamma \frac{f^{\prime \prime \prime}(R)}{f^{\prime \prime}(R)}+\frac{\partial \gamma}{\partial R}=0,
\end{aligned}
$$

where the following constraint has to be satisfied

$$
\begin{aligned}
& \frac{1}{2} r^{2}\left(f(R)-R f^{\prime}(R)\right)(\alpha+\beta)-f^{\prime}(R)(\alpha+\beta)-r^{2} R f^{\prime \prime}(R) \gamma-2 f^{\prime \prime}(R) \gamma+e^{-\lambda}\left[f^{\prime}(R)(\alpha-\beta)+\right. \\
& \left.-r \lambda_{r} f^{\prime}(R)(\alpha-\beta)-2 r \lambda_{r} f^{\prime}(R) \frac{\partial \beta}{\partial \lambda}+2 \gamma f^{\prime \prime}(R)\left(1-r \lambda_{r}\right)\right]=0 .
\end{aligned}
$$

If we consider that $f^{\prime \prime}(R) \neq 0$, that is the trivial solution $f(R)=R$ is excluded, Eqs. (22)-(26) and (28)-(301) can be immediately solved giving the result

$$
\alpha=\alpha(\nu), \quad \beta=\beta(\lambda), \quad \gamma=\gamma(R) .
$$

Eq. (27) can be recast in the form

$$
\frac{\alpha}{2}+\frac{d \alpha}{d \nu}-\frac{1}{2} \beta=-\frac{f^{\prime \prime \prime}(R)}{f^{\prime \prime}(R)} \gamma-\frac{d \gamma}{d R}=\mu_{0}
$$


where $\mu_{0}$ is a constant, because the r.h.s. of the first equality depends only on $R$ while the l.h.s. one is a function of $\nu$ and $\lambda$, which are independent variables. We can assume $\mu_{0}=0$, so we obtain, from Eq. (34) the following set of equations:

$$
\begin{gathered}
\frac{\alpha}{2}+\frac{d \alpha}{d \nu}=\frac{1}{2} \beta, \\
\frac{d \gamma}{\gamma}=-\frac{f^{\prime \prime \prime}(R)}{f^{\prime \prime}(R)} d R .
\end{gathered}
$$

By the same arguments used above, we obtain, from Eq. (35), that $\beta$ is constant, and using this result and putting Eq. (36) into Eq. (31), we get

$$
\alpha=\beta=\alpha_{0},
$$

where $\alpha_{0}$ is a constant, being this result consistent with Eq. (35). Moreover, integrating Eq. (36) we obtain

$$
\gamma=\frac{\mu_{1}}{f^{\prime \prime}(R)}
$$

being $\mu_{1}$ an integration constant. Now the constraint (32) becomes

$$
\alpha_{0}\left(f(R)-R f^{\prime}(R)\right) r^{2}-2 \alpha_{0} f^{\prime}(R)-\mu_{1} r^{2} R-2 \mu_{1}+2 \mu_{1} e^{-\lambda}\left(1-r \lambda_{r}\right)=0 .
$$

Using Eq. (20) for the conservation of the current $j^{\mu}$, we immediately obtain

$$
\partial_{r}\left[\left(r^{2} e^{\frac{\nu-\lambda}{2}}\right)\left(\alpha_{0} f^{\prime \prime}(R) R_{r}+\alpha_{0} \frac{2}{r} f^{\prime}(R)+\mu_{1} \nu_{r}\right)\right]=\partial_{t}\left[\left(r^{2} e^{\frac{\lambda-\nu}{2}}\right)\left(\alpha_{0} f^{\prime \prime}(R) R_{t}+\mu_{1} \lambda_{t}\right)\right] .
$$

For the particular choice $\alpha_{0}=0$, the constraint (39) becomes

$$
R=-\frac{2}{r^{2}}+e^{-\lambda}\left(\frac{2}{r^{2}}-\frac{2}{r} \lambda_{r}\right)
$$

while Eq. (40) is

$$
\partial_{r}\left(r^{2} e^{\frac{\nu-\lambda}{2}} \nu_{r}\right)=\partial_{t}\left(r^{2} e^{\frac{\lambda-\nu}{2}} \lambda_{t}\right)
$$

Using Eq. (42) into the expression for the Ricci scalar (3), it is immediate to verify that the dynamical constraint (41) is satisfied. In this way we have that our system of equations is consistent having the four equations (77), (8), (41) and (42), in the four unknown functions $\nu(r, t), \lambda(r, t), R(r, t)$ and $f(R)$. Our task is now to determine these unknown functions selected by the presence of the Noether symmetry.

\section{EXACT SOLUTIONS}

We are able to solve the dynamics by the Noether symmetry approach. Considering Eqs. (7), (8) and (41), in the case of constant curvature $R=R_{0}$, we obtain the following solutions for the metric coefficients

$$
e^{\lambda(r, t)}=\frac{1}{1+\frac{R_{0} r^{2}}{6}-\frac{B_{1}(t)}{2 r}}=B_{2}(t) e^{-\nu(r, t)}
$$


where $B_{1}(t)$ and $B_{2}(t)$ are integration functions. Moreover we get as constraint $f\left(R_{0}\right)=0$. Then Eq. (42) for the conserved current, using the solution (43) with $B_{1}(t)=B_{1}=$ constant, gives

$$
R_{0}=0
$$

Finally, using this result in Eq. (43) and rescaling the time as in Eq. (13), we obtain the following time-independent solution

$$
d s^{2}=\left(1-\frac{B_{1}}{2 r}\right) d \tilde{t}^{2}-\frac{1}{\left(1-\frac{B_{1}}{2 r}\right)} d r^{2}-r^{2} d \Omega^{2},
$$

which is the well-known Schwarzschild solution in GR. We can conclude that Noether Symmetry Approach for $f(R)$ gravity reproduces the same solutions of GR in the case of constant curvature. This is not a new result but it is useful to test the method [17].

However, other interesting solutions can be found in the case of non-constant curvature in a spherically symmetric static metric. In fact, the current conservation Eq. (42) becomes now

$$
\frac{d}{d r}\left(r^{2} e^{\frac{\nu-\lambda}{2}} \nu_{r}\right)=0
$$

where $\nu=\nu(r)$ and $\lambda=\lambda(r)$. Assuming, as standard, that $\nu=-\lambda$ [33], Eq. (46) can be easily solved to give

$$
\nu(r)=\ln \left(C_{1}-\frac{1}{r}\right)+C_{2}=-\lambda(r),
$$

being $C_{1}$ and $C_{2}$ integration constants. Subtracting Eqs. (7) and (8), after some algebra, we obtain

$$
f^{\prime}(R(r))=D_{1} r+D_{2}
$$

where, again, $D_{1}$ and $D_{2}$ are integration constants. In order to find the form of the $f(R)$-model, we need to express $f^{\prime}(R)$ as an explicit function of $R$. Then using the expression for the Ricci scalar (41), which now is

$$
R(r)=2 \frac{\left(C_{1} e^{-C_{2}}-1\right)}{r^{2}},
$$

we obtain $r$ as a function of $R$. Substituting into Eq. (48), we are able to perform the integration obtaining

$$
f(R)=2 D_{1}\left(2 C_{1} e^{-C_{2}}-2\right)^{\frac{1}{2}} R^{\frac{1}{2}}+D_{2} R+D_{3},
$$

where $D_{3}$ is a constant. It can be demonstrated that our system of equations, considering also Eq. (8), is solved for given choices of the constants. For $C_{1}=\frac{1}{2}, C_{2}=0, D_{2}=-3 D_{1}$ and $D_{3}=0$, we get the metric

$$
d s^{2}=\left(\frac{1}{2}-\frac{1}{r}\right) d t^{2}-\frac{1}{\left(\frac{1}{2}-\frac{1}{r}\right)} d r^{2}-r^{2} d \Omega^{2} .
$$

The selected $f(R)$-function is

$$
f(R)=D_{2} R-\frac{2}{3} D_{2} \sqrt{-R}
$$

The Ricci scalar is uniquely determined as

$$
R=-\frac{1}{r^{2}}
$$

This solution is physically consistent for $r>0$ and it is asymptotically flat. 


\section{CONCLUSIONS}

In the context of $f(R)$-gravity, a Lagrangian approach has been developed to study dynamics of spherically symmetric metrics. We have obtained the Euler-Lagrange equations and solved them in the case of constant curvature $R=R_{0}$ recovering the standard Schwarzschild-de Sitter solution of GR. In this case, the Birkhoff theorem holds. Then the Noether Symmetry Approach has been developed in order to reduce dynamics by finding out conserved quantities, which can be expressed as currents. After, we have solved the Noether system related to the condition $L_{\mathbf{X}} \mathcal{L}=0$, deriving a constraint equation over the dynamics and a conserved current expressed as functions of the dynamical variables. The constraint allows to select the form of the $f(R)$-model. From this point of view the Noether symmetry ensures the closure of the dynamical system. Finally, we have presented some particular cases showing that Noether symmetries are compatible with GR and that solutions exist also for other $f(R)$-models different from $f(R)=R$. In conclusion, the method reveals a useful approach both to select consistent $f(R)$-models and to find out exact solutions.

[1] R. Utiyama and B. DeWitt, J. Math. Phys 3, 608 (1962).

[2] K.S. Stelle, Phys. Rev. D 16, 953 (1977).

[3] I.L. Buchbinder, S.D. Odintsov, and I.L. Shapiro, Effective Action in Quantum Gravity (IOP, Bristol) (1992).

[4] G. Vilkovisky, Class. Quantum Grav. 9, 985 (1992).

[5] A.G. Riess et al., Astron. J. 116, 1009 (1998).

[6] S. Capozziello, Int. J. Mod. Phys. D 11, 483 (2002).

[7] S. Nojiri and S. D. Odintsov Int. J. Geom. Meth. Mod. Phys. 4, 115 (2007).

[8] S. Capozziello and M. Francaviglia, Gen. Rel. Grav. 40, 357 (2008).

[9] T.P. Sotiriou and V. Faraoni, Rev. Mod. Phys. 82, 451 (2010).

[10] A. De Felice and S. Tsujikawa, Living Rev. Rel. 13, 3 (2010).

[11] M. Di Mauro, L. Fatibene, M. Ferraris, M. Francaviglia, Int. J. Geom. Meth. Mod. Phys. 7, 887 (2010).

[12] L. Fatibene, M. Ferraris, M. Francaviglia, S. Mercadante Int. J. Geom. Meth. Mod. Phys. 7, 899 (2010).

[13] S. Capozziello, V. Faraoni, Beyond Einstein Gravity: A Survey Of Gravitational Theories For Cosmology And Astrophysics, Springer, New York (2011).

[14] S. Capozziello and M. De Laurentis, Invariance Principles and Extended Gravity: Theory and Probes, Nova Science Publishers, New York (2011).

[15] S. Capozziello and M. De Laurentis, Phys. Repts. 509, 167 (2011).

[16] H. Stephani, D. Kramer, M. MacCallum, C. Hoenselaers, and E. Herlt Exact Solutions of Einstein's Field Equations, 2nd edition, Cambridge University Press, Cambridge ( 2003).

[17] S. Capozziello, A. Stabile, A. Troisi, Class. Quant. Grav. 25, 085004 (2008).

[18] V. Faraoni, Phys. Rev. D 81, 044002 (2010).

[19] S. Capozziello, A. Stabile, A. Troisi, Phys. Rev. D 76, 104019 (2007).

[20] T. Multamaki and I. Vilja, Phys. Rev. D 74, 064022 (2006).

[21] T. Multamaki and I. Vilja Phys. Rev. D 76, 064021 (2007).

[22] K. Kainulainen, J. Piilonen, V. Reijonen, and D. Sunhede, Phys. Rev. D 76, 024020 (2007).

[23] T. Clifton and J.D. Barrow Phys. Rev. D 72, 103005 (2005).

[24] R.P. Kerr, Phys. Rev. Lett. 11, 237 (1963).

[25] S. Capozziello, M. De Laurentis, and A. Stabile, Class. Quantum Grav. 27, 165008 (2010).

[26] S. Capozziello and Lambiase G., Gen. Rel. Grav. 32, 295 (2000).

[27] S. Capozziello and A. De Felice, Journal of Cosmology and Astroparticle Physics 08, 016 (2008).

[28] S. Basilakos, M. Tsamparlis, and A. Paliathanasis, Phys. Rev. D 83, 103512 (2011).

[29] L. Sebastiani, S. Zerbini, Eur.Phys.J. C 71, 1591 (2011).

[30] S. Capozziello, J. Matsumoto, S. Nojiri, S. D. Odintsov, Phys. Lett. B 693, 198 (2011)

[31] S. Capozziello, F. Darabi, D. Vernieri, Mod. Phys. Lett. A 26, 65 (2011).

[32] S. Capozziello, R. De Ritis, C. Rubano, and P. Scudellaro Riv. Nuovo Cim. 19 N 4, 1 (1996).

[33] S. Chandrasekhar, The mathematical theory of black holes, Oxford Univ. Press., Oxford (1983).

[34] S. Capozziello, A. Stabile, A. Troisi, Class. Quantum Grav. 24, 2153 (2007). 